Psychosomatische und psychosoziale Medizin spielen eine zunehmend wichtige Rolle im Alltag von allen Ärztinnen und Ärzten. Diese Artikelserie ist entsprechend an Kolleginnen und Kollegen aller Fachrichtungen adressiert. Sie steht im Zusammenhang mit dem Symposium, das unter dem Namen «Rheinfelder Tage» erstmals im November 2003 durchgeführt worden ist.

Die «Rheinfelder Tage» sind entstanden aus dem Wunsch, eine interdisziplinäre Fortbildung anbieten zu können, die sich an Kolleginnen und Kollegen aus allen Fachbereichen richtet, die ihre Kenntnisse auf dem Gebiet der Psychosomatik und der Psychosozialen Medizin erweitern wollen.

Die «Rheinfelder Tage» sind eine gemeinsame Veranstaltung der Akademie für Psychosomatische und Psychosoziale Medizin APPM und der Klinik Schützen Rheinfelden.

Die Symposien sollen der Vertiefung des psychosomatischen Wissens, der Fertigkeiten in Kommunikation und Didaktik dienen. Schlussendlich hoffen die Organisatoren, dass die «Rheinfelder Tage» zur Stärkung der gemeinsamen Identität der psychosomatisch und psychosozial tätigen Ärztinnen und Ärzte beitragen.

Die zweite Tagung ist folgendem Thema gewidmet:

\title{
Psychosomatik und Neurowissenschaften
}

\section{2./23. April 2004 in Rheinfelden}

\section{Donnerstag, 22. April, $20.00 \mathrm{Uhr}$}

Öffentlicher Vortrag mit anschliessender Diskussion

- Die Seele zwischen Neuro- und Geisteswissenschaften Prof. Dr. med. Daniel Hell

\section{Freitag, 23. April 2004, ganzer Tag}

\section{Symposium}

- Die Plastizität des Gehirns und der Entstehungsweg der Psychosomatik Prof. Dr. med. Martha Lehmann-Koukkou, Prof. für Psychophysiologie in der Psychiatrie, Universität Zürich

- Krankheitsbewältigung bei neuroimmunologischen Erkrankungen Prof. Dr. med. Heinz Weiß, Facharzt für Psychotherapeutische Medizin, Chefarzt der Abteilung für Psychosomatische Medizin, Robert-Bosch-Krankenhaus, Stuttgart

\section{Workshops}

- Die Plastizität des Gehirns und der Entstehungsweg der Psychosomatik;

- Krankheitsbewältigung bei neuroimmunologischen Erkrankungen;

- Neurobiologische Grundlagen des chronischen Schmerzsyndroms am Beispiel von Rückenschmerzen;

- Männer/Frauen: neurobiologische Fakten;

- Didaktik/Kommunikation.

Als Fortbildung anerkannt von folgenden Fachgesellschaften: SGIM, SGAM, SNG, SGPP, SGGG, APPM.

\section{Tagungssekretariat}

Klinik Schützen, Bahnhofstrasse 19, CH-4310 Rheinfelden, Tel. 06183626 26, Fax 06183625 11,

E-Mail: rheinfeldertage@klinikschuetzen.ch, Internet: www.rheinfeldertage.ch 